\title{
Théologiques
}

\section{Théologie africaine et vie}

\section{René Heyer et François Kabasele Lumbala}

Volume 19, numéro 1, 2011

Théologie africaine et vie

URI : https://id.erudit.org/iderudit/1014177ar

DOI : https://doi.org/10.7202/1014177ar

Aller au sommaire du numéro

Éditeur(s)

Faculté de théologie et de sciences des religions, Université de Montréal

ISSN

1188-7109 (imprimé)

1492-1413 (numérique)

Découvrir la revue

Citer ce document

Heyer, R. \& Kabasele Lumbala, F. (2011). Théologie africaine et vie.

Théologiques, 19(1), 5-12. https://doi.org/10.7202/1014177ar d'utilisation que vous pouvez consulter en ligne.

https://apropos.erudit.org/fr/usagers/politique-dutilisation/ 


\title{
Théologie africaine et vie
}

\author{
René HEYeR" \\ Théologie morale \\ Université de Strasbourg (France) \\ François Kabasele Lumbala** \\ Théologie des religions \\ Institut catholique de la Méditerranée \\ Marseille (France)
}

Depuis sa création, il y a vingt ans, la revue Théologiques n'avait pas encore eu l'occasion de braquer le projecteur sur l'Afrique. Les recherches en théologie pourtant y foisonnent, que ce soit dans les grands centres universitaires que sont Abidjan, Kinshasa et Yaoundé, pour ne parler que de l'Afrique francophone, ou qu'elles soient le fait de l'importante diaspora étudiante et enseignante de par le monde. Cette remarque, faite au comité scientifique de la revue par son membre strasbourgeois, a été immédiatement retournée par la direction en appel d'offre. C'est ainsi que, dans la suite d'un colloque sur divers aspects de la production et de la formation théologiques africaines tenu à la Faculté de théologie catholique de l'Université de Strasbourg (actes parus dans la Revue des sciences religieuses d'avril 2010), l'initiative d'une recherche thématique sur la vie dans la théologie africaine, uniquement sous forme de propositions d'articles pour Théologiques, a été lancée. Elle s'est trouvée rapidement relayée en plu-

* René Heyer est professeur de théologie morale à la Faculté de théologie catholique de l'Université de Strasbourg. Ses recherches portent sur l'éthique politique, la question des genres, et sur les rapports entre les sciences humaines et la théologie.

* François Kabasele Lumbala est professeur à l'Institut catholique de la Méditerranée (Marseille, France), dans la section de la théologie des religions. Il poursuit actuellement ses recherches sur le phénomène religieux et a écrit de nombreux ouvrages et articles sur les cultures d'Afrique noire, ainsi que sur les rapports entre religions et cultures. Ses publications les plus récentes incluent (2005) Renouer avec ses racines et (2011) Naissances insolites en terre africaine, éditées chez Karthala à Paris. 
sieurs points d'Europe, d'Amérique du Nord et d'Afrique, particulièrement au Congo. Le «local» ou le «continental» africain est ainsi saisi d'emblée dans une dimension mondialisée.

\section{Cheminement de la théologie africaine}

Les théologiens d'Afrique subsaharienne ont conscience de développer une version originale de la théologie et ils ne manquent pas d'être surpris lorsque des Occidentaux déclarent en découvrir l'existence: cela fait plus d'un demi-siècle en effet que cette théologie africaine existe et elle a eu le temps de connaître courants et contre-courants. Le fil conducteur en est la compréhension de l'évangélisation et des conséquences de celle-ci. Après une «théologie de la fondation de la chrétienté » transposant localement, pour les catholiques, l'organisation romaine, sont venus des mouvements de réactions et de reprises qui font place de plus en plus nettement à des valeurs considérées comme spécifiquement africaines (Tchonang, 2010). C'est déjà le cas avec la "théologie de l'adaptation ", amorcée par l'ouvrage collectif Des prêtres noirs s'interrogent (1956). Il s'agissait d'inviter les cultures africaines sur la scène de l'évangélisation. Cette perspective fut encouragée par Paul VI, qui appela à constituer un «christianisme africain» lors d'un déplacement en Ouganda en 1969 (Paul VI, 1969). Mais adapter le message évangélique n'est pas encore reconnaitre son incarnation dans les pratiques et la culture africaines: «l'inculturation» exige de prendre au sérieux une vision du monde distincte de celle où s'est forgée la théologie occidentale. Ces courants théologiques de l'adaptation et de l'inculturation se sont cependant élaborés à une certaine distance de contextes socio-politiques particulièrement préoccupants: Jean-Marc Ela et Engelbert Mveng souligneront parmi bien d'autres la nécessité d'une théologie qui prenne corps dans l'histoire en vue de la libération d'une oppression séculaire, marquée par l'esclavage et la colonisation. Plus récemment, des théologiens tels que les protestants Jesse Mugambi et Kä Mana ont appelé à une refonte des habitudes de pensée par une «théologie de la reconstruction" qui doit être globale à leurs yeux; elle passe par la reprise en mains de leur destin par les Africains eux-mêmes et est censée modifier jusqu'à leur imaginaire.

Une précision: si l'on a pu parler de théologies de la libération en Afrique, c'est dans un sens nettement différent des théologies de l'Amérique latine. Sur le continent américain, la libération du peuple opprimé passait nécessairement par le renversement des structures économiques et 
la redistribution des terres; en Afrique postcoloniale, la libération commençait par des efforts de recouvrement d'une identité niée et gommée, efforts de désaliénation, efforts d'une "décolonisation mentale» (Mabika Kalanda, 1967); c'est pour cela que l'accent culturel a semblé prédominer dans cette libération. Mais du reste, le culturel a toujours englobé le politique, du moment que toute politique se dessine au sein d'une culture.

Dans un souci de respect du pluralisme, on a également voulu parler de théologies africaines au pluriel (comme on parle «des» Afriques). Un théologien moraliste comme Bénézet Bujo (2008) récuse cette diversité de surface. Il part précisément de l'existence du «christianisme africain », qui est certes bigarré mais indéniablement reconnaissable dans ses valeurs et son expression: «la » théologie africaine est la théologie de ce christianisme-là. C'est donc depuis l'épaisseur d'une expérience profuse qu'on pourra percevoir les traits propres d'une théologie qui forment aussi son apport à la vie chrétienne et à la pensée d'autres régions du monde. Certains de ces traits ont été reconnus par l'Église romaine: ainsi le sens africain de la famille, avec ses valeurs de solidarité, d'accueil, de chaleur des relations par-delà tout ethnocentrisme, a été consacré lors du synode de 1994 pour l'Afrique avec la notion d' "Église famille de Dieu» (JeanPaul II, 1995).

Mais le thème le plus important peut-être de la théologie africaine, relevé depuis Placide Tempels et sa Philosophie bantoue (1949), est celui de la vie (Benoît XVI, 2011). Le rôle majeur joué par la vie n'a rien d'étonnant, à première vue: quoi de plus immédiatement saisissable pour tout vivant que sa vie? En ce sens, on comprend bien comment la théologie africaine est d'abord une théologie de la vie en plénitude. En un autre sens, la vie est ce qui déjoue l'appréhension, qu'elle soit physique ou métaphysique, et elle apparaît alors, en tant qu'emblème de la pensée africaine, comme un coin enfoncé dans l'épistémologie et l'ontologie occidentales.

On se rappelle en effet le célèbre constat du biologiste François Jacob: "On n'interroge plus la vie aujourd'hui dans les laboratoires [...]. On s'efforce seulement d'analyser des systèmes vivants» (Jacob, 1970, 320-321). Savoir ce qu'est la vie apparaît aux scientifiques une question générale à laquelle ils ne cherchent plus à apporter de réponse. Invoquer la vie, seraitce alors verser dans le vitalisme? Mais on se souviendra que le vitalisme naît dans les parages de la science moderne et qu'il désigne (contre l'âme) la matière du vivant. La promotion de la vie en Afrique n'est pas réductible à cette forme de matérialisme. La tradition occidentale de la philosophie pour sa part, axée sur une pensée de l'être, a du mal à saisir la vie autre- 
ment que comme ce qui émerge. La vie émerge, elle est émergence, mais comment relier son organisation générale et ses manifestations singulières? Il ne serait pas possible en somme de remonter à propos de la vie au-delà de la compréhension pré-conceptuelle que nous en avons tous (et dont procèderait la philosophie elle-même). Demeure cependant le fait que la vie est un absolu (un absolu sans contraire: la vie ne meurt pas, seuls meurent les vivants). Or cet absolu nous émeut: "C’est peut-être une des raisons de la résonance en chacun de l'immémorial» (Courtel, 1999, 407).

On attendra certainement de l'Afrique, du christianisme qui s'y développe et de sa théologie, un témoignage de cette résonance de l'immémorial. Mais on sera également attentif, face à l'extériorité qui a fini par caractériser la pensée de l'être en Occident, à l'élévation de la vie (du sentiment intime, communautaire et cosmique de la vie) au rang de concurrent de l'être.

\section{De l'amour de la vie à la rencontre du Vivant}

Les Africains aiment et célèbrent la vie. Pour eux, la vie est le bien suprême, le don sacré par excellence de l'Au-delà. Au cours des péripéties atroces de leur histoire, beaucoup d'entre eux ont préféré demeurer dans une vie d'esclave plutôt que de se donner la mort, devant la négation de leur liberté! On peut critiquer un tel amour de la vie. Mais c'est un choix qui répond à une conception du monde et de la vie partagée par la plupart des peuples d'Afrique noire subsaharienne. Vivre, pour eux, c'est avoir de la force, être en bons rapports avec Dieu et les ancêtres qui sont respectivement la source et le canal par où leur vient la vie, être en harmonie avec les vivants terrestres et la nature qui les entoure et dont ils sont un maillon de la vie; vivre, pour eux, c'est transmettre et donner la vie qu'ils ont reçue...

Les contributions qui suivent ont exploré différents aspects de cet amour pour la vie en Afrique noire: Nathanaël Yaovi Soede constate que les communautés d'Afrique subsaharienne mesurent et se donnent le temps en fonction des dimensions de leur vie; Ghislain Tshikendwa Matadi montre que la vie pour les Africains vaut plus que toutes les souffrances qu'on peut y rencontrer; Ntumba Muena Muanza et Lupwishi Mbuyamba explorent le dynamisme de cette vie particulièrement dans l'art du récit et des contes, dans les techniques de l'oralité, débusquant ainsi le pouvoir social, économique et politique de l'art; tandis que Marcel Anganga remonte à l'amont, pour retrouver ce dynamisme et cet amour de la vie dans les traditions de l'Égypte ancienne, Lambert Museka Ntumba les détecte simplement aujourd'hui dans la vie quotidienne des chrétiens afri- 
cains ainsi que dans les luttes actuelles pour la vie dans nos communautés, de manière à laisser surgir la question de savoir si la vie recherchée en Afrique est celle qu'évoque Jésus dans l'Évangile. François Kabasele Lumbala explore les célébrations chrétiennes africaines pour y découvrir et mettre en lumière cet amour de la vie; dans son analyse de théologien africain, Didier Mupaya Kapiten fait apparaître, dans les initiations africaines traditionnelles, des paradigmes du sacrifice du Christ qui passe par la mort pour faire épanouir la vie; pour Adrien Ntabona, l'amour pour la vie chez les Africains inspire la restructuration et le fonctionnement des nouvelles communautés chrétiennes au sein des paroisses, en vue de passer du communautarisme traditionnel à la communion de foi. Mais l'éclairage sur la vie a dû emprunter également des points de vue venus d'ailleurs, car il s'agit de la même vie humaine; aussi René Heyer a-t-il réagi face à certaines analyses et à une certaine terminologie ( "paupérisation anthropologique»!) en défendant la vie; quant aux étudiants de l'École doctorale de théologie et de sciences religieuses de Strasbourg, qui ont pu lire les articles du dossier lors de sa préparation, dans le cadre de leur séminaire, ils ont attiré l'attention sur le danger d'une idéalisation facile et abusive d'une Afrique révolue.

De ces différentes contributions, des convergences ressortent. D’abord par rapport à la vie, la réflexion philosophique et théologique du dossier tend vers l'unanimité: Dieu est le vivant par excellence, la «plénitude-vie », la "totalité-vie », d'où toute vie tire sa source et où elle se ressource; la vie et la mort sont intimement liées, au point que sans la mort, la vie ne serait plus la vie; aussi l'initiation africaine est-elle l'école par excellence où se fait l'apprentissage du passage par la mort pour le renforcement de la vie, et le triomphe de la vie sur la mort, but poursuivi par toute l'Afrique; enfin, nos auteurs soulignent que le désir de vivre et la joie de vivre est une disposition qui rend l'Africain ouvert à la rencontre avec Jésus Christ, et que cette disposition est un appel à l'accomplissement, un appel à devenir des artisans de l'histoire.

Ensuite au plan historique et sociologique, une convergence se dessine dans la mise à jour du rôle dévastateur de la colonisation et d'une certaine pastorale de la terre brûlée; la question de la pauvreté actuelle de l'Afrique est abordée et attribuée tant à la domination par l' "Euramérique » qu'au manque d'initiatives des Africains eux-mêmes; la plupart des contributions soulignent la nécessité pour l'Afrique de sortir d'un régime d'imitations serviles et caricaturales, pour créer et progresser à partir de ses propres racines; c'est ainsi que d'aucuns reviennent sur le lien entre 
l'Égypte pharaonique et l'Afrique noire, dans le but d'y trouver des fondements solides pour une renaissance en Afrique d'aujourd'hui.

Pour arriver à serrer un sujet si englobant, "la vie », l'interdisciplinarité s'est imposée: il a fallu recourir à différents éclairages, des torches de l'histoire, des lampes de la sociologie, de la lumière de la philosophie, des rayons de la pédagogie et de l'esthétique; et même au sein de la théologie africaine, le point de vue de la pastorale de la vie en appelait à celui de la dogmatique; la célébration de la vie doit s'appuyer sur l'histoire des rites et emprunter des éléments tant à la psychologie qu'à l'anthropologie des rites; au long des différentes questions et réflexions, nous avons senti que les sciences du développement et l'économie avaient un mot à dire sur l'utilisation de telle ou telle forme d'organisation des communautés chrétiennes, pour mieux s'assurer du triomphe de la vie sur la mort.

Mais revenons à la l'élaboration théologique africaine de la vie. Quand on fait de la théologie dans un contexte où la vie est recherchée comme le don sacré par excellence, les paroles de Jésus en rapport avec la vie ne peuvent passer inaperçues; elles sont au contraire d'emblée mises sur un piédestal: «Je suis venu vous apporter la vie, et je vous l'apporte abondante» (Jn 10,10b). Cette vie, Jésus l'apporte de chez son Père qui en est la source, et elle passe par la foi en Jésus: "quiconque voit le Fils et croit en lui, a la vie éternelle» (Jn 6,40). L'évangile de Jean, à ce sujet, paraît être un développement particulier de cette veine de la vie divine. Mais qu'est-ce que la vie divine qu'apporte Jésus? C'est peut-être sur ce point que les différentes contributions ne se sont pas attardées, et il serait utile d'y apporter quelques précisions.

Est-ce un surplus ajouté à la vie humaine, une sorte de «sur-humanité »? Non; c'est le lieu de l'accomplissement dans la vie humaine: nos soifs d'eau et nos faims de nourriture sont assouvies dans la vie divine (la Samaritaine, la foule rassasiée de pain après la multiplication)! Nous connaissons un renouvellement de vie qui ne s'épuise pas, nous vieillissons sans vieillir (Nicodème), nous mourons sans cesser de vivre (à Marthe et Marie dont Jésus ressuscite le frère). La vie dont le Christ a le secret est l'accomplissement d'une plénitude vitale qui nous fait participer à la joie divine d'exister, à la communion avec le Père, à travers le Fils, comme les sarments sont en communion avec la vigne (Jn 15,4-6). Plus le lien entre les disciples et Jésus est fort, plus le disciple est vivant. Finalement, vivre de la vie divine, c'est vivre au cœur même de la communion trinitaire, une communion qui nous ouvre à tous nos frères: «La vie éternelle, c'est qu'ils te connaissent toi, le seul véritable Dieu et celui que tu as envoyé, JésusChrist» (Jn 17,3); "Si quelqu'un a soif, qu'il vienne à moi et qu'il boive. 
Celui qui croit en moi [...] de son sein couleront des fleuves d'eau vive [...]. Il parlait de l'Esprit que devraient recevoir ceux qui avaient cru en lui [...]» (Jn 7,37-39). L'Esprit jaillit du sacrifice de la vie. L'Esprit Saint, c'est le souffle de cette vie divine qui culmine dans le don de soi, dans le sacrifice consenti pour les autres.

Voilà pourquoi les Africains se sont particulièrement réjouis à la rencontre avec le christianisme; ils ont cru y trouver des éléments de renforcement de leur vie. Tout comme ils avaient accueilli les colons occidentaux avec leur sel de mer, leurs méthodes de transformation des métaux, leurs techniques de filature, leurs écritures, leurs langues, dans l'espoir que leur vie se renforcerait, ainsi ont-ils aussi accueilli le message du Christ. Mais un accueil n'est pas une annihilation de soi-même, ni de son identité. Un accueil est une disposition à l'ouverture, en vue d'un progrès; c'est cela qui apparaît dans les propos de théologiens africains; c'est aussi cela qui leur est rappelé sous diverses formes par les critiques de leurs confrères et collègues, étrangers ou africains. Toutes les cultures ont des tares qu'il faut corriger à la lumière de l'Évangile. Mais toutes les particularités raciales demeurent une chance pour l'humanité et le christianisme lui-même. Aussi l'inculturation du message chrétien dans les différentes cultures est-elle la seule voie d'évangélisation qui rende compte de la richesse infinie et inépuisable du divin, et ce, malgré les dérapages inhérents à tout cheminement d'inculturation.

Loin d'épuiser les dimensions de la vie et ses ramifications dans les tissus culturels de l'Afrique noire d'aujourd'hui, ces réflexions constituent une amorce de plus amples développements, des perspectives à prolonger, des terrains à défricher. Car il y a bien de questions soulevées et qui demeurent, dont celles qui suivent: Quelle est la contribution de l'Afrique noire au triomphe de la vie sur la terre? Suffit-il d'invoquer l'amour de la vie, les mythes et dictons sur la mort et la vie, de clamer l'Afrique «mère de la vie »? Quelle est la place de l'Afrique dans l'Église chrétienne, dans le monde actuel? Le christianisme est-il exogène à l'Afrique? Quelle part de vie et de mort est-elle attribuable à la colonisation et au christianisme en Afrique? Comment peut-on assurer la continuité des traditions et cultures africaines, tout en la conjuguant aux besoins de progrès? Comment inscrire aujourd'hui une personnalité artistique dans le cadre d'une identité africaine? Comment conjuguer créativité et inventivité avec le renouvellement des cultures dans l'évolution vertigineuse de ce monde? Comment se fait-il que dans une Afrique qui aime la vie, il y ait tant d'atrocités et de carnages contre la vie, tant de violations massives des droits humains? 
Même si des questions demeurent en suspens, l'apport de ces contributions saute aux yeux. D'abord, elles nous éclairent et donnent des précisions utiles sur «mort et vie» dans les cultures d'Afrique noire. Ensuite, elles mettent à notre disposition des éléments d'analyse et de réflexion: dans un monde où la mort tend à être cachée, évacuée de la vie courante, les expériences initiatiques d'Afrique font entendre l'appel à vivre notre mort pour le renforcement de notre vie, dans le renoncement à soi-même, dans l'assomption des contraintes de la vie, dans les sacrifices consentis pour le bonheur de tous. Enfin elles tentent de nous persuader que, quelles que soient nos idéologies politiques et anthropologiques, la vie sera sauvegardée sur la terre si l'être humain reste premier, et que «le temps est fait pour l'être humain et non l'être humain pour le temps ", pour un temps conçu en termes d'intérêt et d'argent.

\section{Références}

BENô̂t XVI (2011), Africae munus. Exhortation apostolique post-synodale sur l'Église en Afrique au service de la réconciliation, de la justice et de la paix, Vatican/Kinshasa, Librairie vaticane/Médiaspaul.

Bujo, B. (2008), Introduction à la théologie africaine, Fribourg (Suisse), Presses universitaires.

Collectif (1956), Des prêtres noirs s'interrogent, Paris, Cerf.

Courtel, Y. (1999), "La vie retrouvée», Revue des sciences religieuses 73/4: «Approches de la vie», p. 405-424.

Јасов, F. (1970), La Logique du vivant. Une histoire de l'hérédité, Paris, Gallimard.

Jean-Paul II (1995), Ecclesia in Africa. Exhortation apostolique postsynodale sur l'Église en Afrique et sa mission évangélisatrice vers l'an 2000, Kinshasa, Médiaspaul.

Mabika-Kalanda (1967), La remise en question, base de la décolonisation mentale, Bruxelles, Remarques africaines (Études congolaises 4).

Paul VI (1969), "Allocution au symposium des évêques d'Afrique et de Madagascar ", Documentation catholique n 1546, p. 763-765.

Tchonang, G. (2010), "Brève histoire de la théologie africaine », Revue des sciences religieuses 84/2: "Théologies africaines", p. 175-190.

Tempels, P. (1949), La philosophie bantoue / trad. par A. Rubbens, Paris, Éditions africaines (Présence africaine). 